\title{
Optical Measurement of Bubbles: System Design and Application
}

\author{
IRA LEIFER \\ Chemical Engineering Department, University of California, Santa Barbara, Santa Barbara, California \\ GERRIT DE LEeuw AND LeO H. COHEN \\ TNO Physics and Electronics Laboratory, The Hague, Netherlands
}

(Manuscript received 29 November 2001, in final form 12 February 2003)

\author{
ABSTRACT
}

\begin{abstract}
Affordable high quality charge-coupled device (CCD) video cameras and image processing software are powerful tools for bubble measurements. Because of the wide variation between bubble populations, different bubble measurement systems (BMSs) are required depending upon the application. Two BMSs are described: a mini-BMS designed to observe the background bubble population from breaking waves, and a large-BMS designed to noninvasively determine the time-resolved bubble distribution inside dense bubble plumes and near the interface, as are details of the analysis techniques. Using the two systems in conjunction with each other allowed size distributions over the range $15-5000-\mu \mathrm{m}$ radius to be obtained.

The BMSs were designed for application to breaking-wave bubble plumes in the field or laboratory. Distributions measured by both BMSs in aerator-generated plumes agreed very well for the overlapping size range. Also presented are observations of bubble plumes produced by breaking waves in a large wind-wave flume, and calibration experiments showing the effect on measured bubble size due to blur induced by slow shutter speeds.
\end{abstract}

\section{Introduction}

Bubbles are important to many processes of interest in geophysics (Farmer et al. 1993; Asher et al. 1996; Woolf 1997), as well as in other disciplines such as chemical engineering (Clift et al. 1978), biopharmaceuticals (Kawase et al. 1992), and waste-water management (Asselin et al. 1998), among many others. Within geophysics, bubble plumes from breaking waves are either dominant or very important to processes as diverse as air-sea gas transfer (Liss et al. 1997), marine aerosol formation (Blanchard 1963; Monahan 1986; De Leeuw 1990; Spiel 1998), surface microlayer enrichment (Blanchard 1989), and turbulence generation (Thorpe 1982). Each of these processes has potentially important impacts on both global and regional scales.

Bubbles both directly and indirectly enhance atmosphere-ocean exchange of gases that are important to global climate, such as methane, carbon dioxide, and fluorocarbons. Bubbles directly exchange entrained atmospheric gas with the ocean by diffusion of the gas

Corresponding author address: Dr. Ira Leifer, Marine Sciences Institute and Chemical Engineering, Chemical Engineering Dept., Bldg. 2, Rm. 3357, University of California, Santa Barbara, Santa Barbara, CA 93106-5080.

E-mail: ira.leifer@bubbleology.com. through the bubble-ocean interface. Indirectly they contribute to gas exchange by disrupting the surface microlayer (Kitaigorodskii 1984), by generating turbulence as they rise in the upper ocean (Thorpe 1982), and by the release of entrained gas when they burst at the surface (Liss et al. 1997). Additionally, bubble plume formation (i.e., wave breaking) is inextricably linked with turbulence generation. These turbulence formation mechanisms alter the turbulent velocity profile within the oceanic boundary layer, thereby enhancing gas transfer (Kitaigorodskii 1984). Bubble plumes also cause bulk fluid motions, such as an upwelling flow (Asher et al. 1997), enhancing mixing and gas transfer.

Due to their importance, the development of bubble measurement systems (BMSs) to determine the bubble size distribution (number of bubbles per unit volume per size increment) has long interested researchers. Two optical BMSs were developed and used to measure bubbles in the background and plume populations during air-sea gas transfer experiments in a laboratory situation (De Leeuw et al. 1999) and for later oceanic deployment. In this paper we present a review of previous bubble measurement systems. Following, a discussion of the design criteria and a detailed description of the two systems is provided. Also presented are some results from an intercalibration experiment and bubble observations during the LUMINY experiment. 


\section{a. Previous bubble measurement systems}

Different approaches have been used to measure bubbles, from acoustics to optics (laser and video/photo). Each technique has advantages and disadvantages and is effective over different size ranges and bubble density regimes. Laser techniques are noninvasive and use a laser beam that has its illumination blocked when a bubble crosses the narrow measurement volume (Hwang et al. 1990), or multiple beams whose intersection defines the measurement volume. The bubble size is determined by its interaction with the laser interference pattern (Baldy and Bourguel 1987; Asher and Farley 1995). Laser systems have problems when multiple bubbles enter the beam(s) - that is, high bubble density results in coincidence-and with large $(r>1000 \mu \mathrm{m}$, where $r$ is radius) bubbles whose mean shape is ellipsoidal and variable due to shape oscillations. The shape of large bubbles may also cause aliasing wherein large bubbles are sized as smaller bubbles (Asher and Farley 1995). Since bubble concentration typically decreases with $r^{-2}$ to $r^{-4}$, (e.g., De Leeuw and Cohen 1994; Haines and Johnson 1995), aliasing only significantly (under) biases large bubble concentrations. Acoustic approaches include resonance, pulse propagation, or multifrequency backscatter. Acoustic methods have high rejection of nonbubble particles, for example, $10^{-9}$ for acoustic resonators (Vagle and Farmer 1998), and can noninvasively map large regions of the water column, allowing investigations into bubble plume distributions (Thorpe 1982; Medwin and Breitz 1989). However, acoustical methods have difficulties, in common with laser methods, with nonspherical (i.e., $r>500 \mu \mathrm{m}$ in a turbulence flow) bubbles and at high bubble concentrations. Optical methods (photo and video) are able to measure at high bubble densities and over a wide $r$ range including very large bubbles (the optics determines the size range). Optical approaches use either a constrained measurement volume (e.g., Monahan et al. 1994; Wang and Monahan 1995) or an unconfined measurement volume (e.g., Johnson and Cooke 1979; Walsh and Mulhearn 1987; Jähne and Geißler 1994; Haines and Johnson 1995; Deane and Stokes 1999). Optical systems are generally invasive since for clarity in turbid water, or for high bubble concentrations, the measurement volume must be near the system's viewing port. However, invasive systems may significantly disturb the bubbly flow.

Bubbles are typically backlit, although side and front lighting (discussed below) have been used. The collected images are analyzed and the number (or probability) of bubbles in each size class determined. The bubble concentration distribution, based on the measurement volume, can be calculated from the probability distribution. The measurement volume for constrained systems varies only with the effect of bubbles touching the image edge (i.e., a large bubble must be farther from the edge than a small one to avoid its images being partially cut), and very large bubbles whose size approaches that of the measurement volume, and thus have difficulty entering. In contrast, one of the major disadvantages of an unconstrained optical BMS is the difficulty in determining its measurement volume, since the depth of field varies with bubble size, optics, and the analysis method. Furthermore, the measurement volume may vary with illumination and water clarity.

In optical systems, apparent bubble size varies with analysis technique (e.g., the threshold level, image preprocessing, etc.) and distance to the bubble unless telecentric lenses are used (only feasible for longer focal lengths and thus higher magnification). Johnson and Cooke (1979) used three forward-pointing strobes. Bubbles were "in focus" when the three spots did not overlap, and the measurement volume was a simple function of $r$ up to the large $r$ limit of $\sim 300 \mu \mathrm{m}$. Walsh and Mulhearn (1987) used a similar approach and found that the spot location on the bubble is complicated by optical effects due to refraction and reflection on the curved bubble surface. They estimated a sizing error of $\sim 10 \%$. Haines and Johnson (1995) side-illuminated bubbles in a 5-cm-thick slab. For bubbles in this light slab, the size error was determined by moving a bubble attached to a wire along the camera axis, and was $\sim 10 \%$ across the light slab for a $r=250 \mu \mathrm{m}$ bubble.

Constrained systems also may have the problem of evolution of the size distribution between entry into the device and reaching the measurement volume since bubble rise velocity increases with $r$. If this distance is short, segregation is negligible. An extreme example is a bubble tube where bubbles enter and rise through a clear tube above the water surface for imaging. The entry distribution can be calculated using the bubble rise velocity (Bowyer 1992). Since bubble rise velocity varies with surfactants (Clift et al. 1978), where too narrow, and walls (Uno and Kintner 1956), the rise velocity should be measured.

\section{b. Design considerations}

Most bubbles produced by breaking waves rapidly return to the surface due to the buoyancy force. However, some bubbles, particularly smaller ones, diffuse out of the plume into a steady-state background population, whose size and depth distribution primarily is determined by turbulence diffusion (Thorpe 1982). Most published bubble distributions are time- and spatially averaged measurements (e.g., Baldy 1988; Medwin and Breitz 1989; Farmer et al. 1993; De Leeuw and Cohen 1994; Asher et al. 1996) at a significant depth below the surface and thus are heavily biased toward the background population. Such distributions greatly underestimate the total initial population (total number of bubbles per size unit produced), since a common feature of all these time-resolved distributions was a steepening of the distribution with time as the larger, faster rising bubbles reached the surface earlier. The few published 
time-resolved (but spatially averaged) distributions have been for tipping bucket experiments (Bowyer 1992; Monahan et al. 1994; Leifer 1995; Haines and Johnson 1995).

The majority of large bubbles produced by a breaking wave do not diffuse into the background population but are confined in a dense and coherent bubble cloud or plume. Within the plume, large bubble concentrations can be several orders of magnitude greater than in the background population. Additionally, the initial bubble size distribution varies weakly with $r$ (e.g., Haines and Johnson 1995), while time-averaged distributions typically vary strongly with $r$. As a result, time- and spatially averaged distributions are likely to underestimate significantly the relative importance of large versus small bubbles. This is not to say that time- and spatially averaged distributions are unimportant; for the transfer of very poorly soluble gases, sound scattering and generation, and potentially aerosol generation, the background distribution is relevant or even dominant. However, it is important to measure the appropriate population depending upon the process of interest.

These ideas were investigated with bubble measurements during the LUMINY experiments in the large airsea interaction simulation tunnel of the Laboratoire Interactions Océan-Atmosphère LUMINY, Institut de Recherche sur les Phénomènes Hors Equilibre (IRPHEIOA) in Marseilles, France, hereafter referred to as LUMINY. The LUMINY experiment is described elsewhere (De Leeuw et al. 1999, 2001). In brief, distributions of bubble plumes created by aeration devices (a submerged grid of porous ceramic tubes) as well as by wind stress-induced wave breaking were measured. Wave development could also be increased by a submerged paddle wavemaker.

\section{Bubble measurement systems}

A large-bubble measurement system (large-BMS) was developed at the National University of Ireland, Galway (NUIG), and a mini-bubble measurement system (mini-BMS) was developed at TNO Physics and Electronics Laboratory in The Hague, Netherlands, for deployment during LUMINY to quantify the breakingwave bubble distribution. These two optical systems were quite different and provided complementary information regarding the bubble sizes and processes that could be studied, allowing characterization of both the background and plume bubble populations. The miniBMS observed bubbles ranging from $15<r<500 \mu \mathrm{m}$ within a well-defined sample volume. The latter allowed calibration of the bubble concentration but prevented observation near the interface (where the support structure produced bubbles), and of larger bubbles (i.e., comparable in size to the sample volume). Thus, the miniBMS was well suited to background distribution characterization. The large-BMS observed bubbles ranging from $r \sim 200 \mu \mathrm{m}$ to the largest bubbles observed, $r \sim$
$5000 \mu \mathrm{m}$, and was designed to minimally interfere in the sample volume. The design allowed measurements near the interface and within the bubble plume, providing bubble plume distributions, and information on plume dynamics. Time- and spatially resolved bubble distributions were determined in bubble plumes, while for the background distributions, time- and spatially averaged distributions were calculated. The two systems were mounted adjacent to each other so neither was upstream of the other's sample volume.

\section{a. Large-bubble measurement system}

The large-BMS used multiple video cameras to allow time-resolved observations simultaneously at multiple resolutions for one or several locations. Components of the large-BMS can be separated into four categories: structural (support framework and housings), imaging (video, electronics, cables, and optics), illumination (lights and diffusion screens), and data acquisition (equipment for recording and analysis). The large-BMS is shown schematically in Fig. 1.

\section{1) StRuCTURAL COMPONENTS}

The structural framework consisted of hollow aluminum tubes mounted below a moveable chariot that allowed the large-BMS to scan fetches in the wind-wave tunnel from 15 to $30 \mathrm{~m}$. Components could be repositioned on the framework allowing observations at different locations by the various cameras. Four video cameras were mounted in individual waterproof aluminum or PVC housings. The housing fronts were a reduced snout into which the lens protruded to minimize flow distortions and bubble production by the camera housing, of particular concern near the interface. Observation was through photographic-quality glass UV filters. The large housings and the diffusive illumination screens interfered with the fluid flow, creating vortices and bubbles. As a result, the sample volume was always far from both screens and housings. Furthermore, the camera and screens were oriented perpendicular to the current and wave direction. Visualization studies showed no effect in the sample volume from the housings and screens. Vortices and generated bubbles were advected downstream.

For BMS observations of dense rising bubble streams, such as can occur from sediments (Leifer et al. 2000a), due to aeration devices or even from a weir (Cipriano and Blanchard 1981), a simple modification of the noninvasive large-BMS was required. Specifically, a simple bubble blocker, shown schematically in Fig. 2, was added that allowed only a fraction of the dense bubble streams to enter the sample volume. All other bubbles were diverted beyond the measurement volume. Furthermore, the blocker did not cause observable coalescence. During LUMINY, the bubble blocker was used only when the aerators were producing bubbles. 


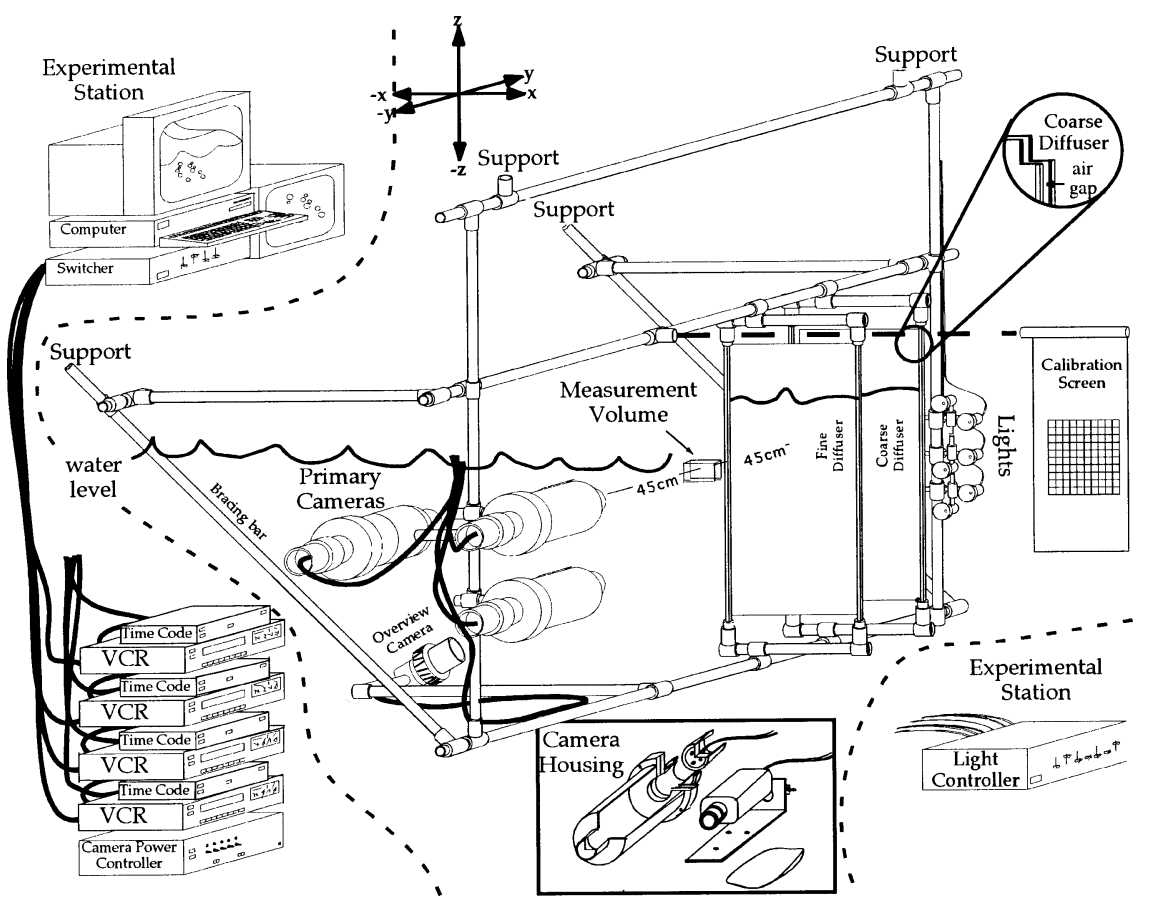

FIG. 1. Large-BMS setup.

\section{2) VIDEO IMAGING}

The large-BMS video subsystem consisted of three primary cameras and one overview camera. The primary cameras were two S-VHS color cameras (JVC, TK 1280) and a monochrome VHS camera (Computar cm510ac, Japan). An assortment of lenses from 12 to $50 \mathrm{~mm}$ was used depending upon the desired field of view (FOV) and resolution. The overview camera was located at approximately $45^{\circ}$ to the primary cameras with a $12-\mathrm{mm}$ wide angle lens. Its depth of field (DOF) allowed everything from a few centimeters to infinity to be in focus.

The chrominance and luminance components of the

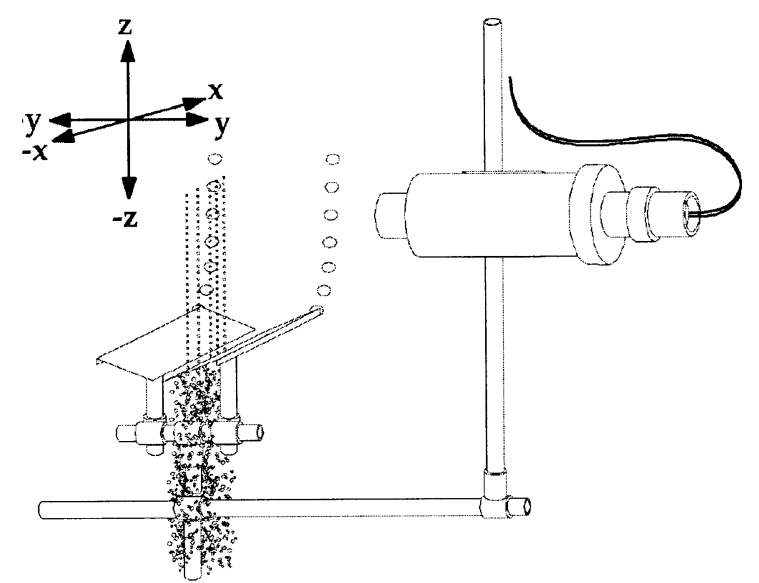

FIG. 2. Schematic of the bubble blocker and orientation to the camera housing. video signals were sent to the experimental station along separate, low-capacitance, 30-m-long BNC cables. Control and power signals were conducted separately along shielded cables. Additional noise reduction was achieved through in-housing regulation for driver circuitry, large $(500 \mu \mathrm{F})$ filtering capacitors on power inputs, and ferrite beads on the AC power lines for the S-VHS cameras. A time code was added to the luminance portion of each signal before recording.

\section{3) ILlumination}

Bubble illumination was by backlighting that caused in-focus bubbles to appear as dark rings with bright interiors. Bubble sizing requires differentiation from the background, but variations in background intensity reduce the contrast, thereby decreasing the image information content in part of the image. It is easy to provide bright, even illumination for an FOV a few centimeters in width; however, to provide even illumination over the larger FOV necessary to study bubble plume dynamics required the use of two diffuser screens. A coarse airfilled diffuser screen scattered light widely over a short distance, decreasing variations between the lamps. A subsequent fine screen, made from sandblasted Plexiglas, removed intensity patterns from the coarse diffuser screen. Due to high bubble velocities and the need for an adequate DOF, fast shutter speeds (1/500-1/1000 s) and small apertures (f4-f8) were used. To provide the requisite intense illumination, six $12-\mathrm{V}, 55-\mathrm{W}$ lamps were used, two for each camera. The lamp intensities were individually variable. 


\section{4) VIDEO ACQUiSITION}

Video signals were recorded by a bank of video tape recorders (VTRs). Two S-VHS VTRs (Panasonic AG4700; Panasonic NV-HS900) were used to record the S-VHS signals, while two VHS VTRs (Mitsubishi) were used to record the VHS primary camera and overview camera. An Apple Power PC 8500 with an internal capture card was used to create digital movies of bubble plumes. The movies typically lasted 1 or $2 \mathrm{~s}$ and were captured from the S-VHS VTR in single field mode, that is, 50 fields per second. Each field was averaged 16 times when captured to reduce noise. The digitization resolution was $568 \times 742$ pixels for a theoretical resolution of $100 \mu \mathrm{m}$ per pixel for a $7.5-\mathrm{cm}$ width FOV. For several reasons, the effective resolution was less. Due to fluid motion, the two video fields are separated by $0.04 \mathrm{~s}$ and cannot be combined, reducing the resolution to $284 \times 742$ pixels. Further losses occur due to optics, water turbidity, high-frequency attenuation in the video cables, and signal degradation on the video tape. Degradation of both the resolution and intensity information decreases the ability to differentiate between the bubble and the background. A reasonable estimate of all these losses is a factor of 2, that is, an effective resolution of $150 \times 375$ pixels, and implies that for reliable identification a bubble must be at least 4 pixels in diameter. A comparison of the measured distribution for two cameras with different FOVs observing the same plume showed a divergence at a radius of $200-250 \mu \mathrm{m}$, or 4-5 pixels, agreeing with this rough estimate of effective resolution. This is discussed in section 4 .

\section{5) Calibration}

For a noninvasive BMS, that is, no physical confinement, the effective measurement volume is difficult to determine, since it is a function of bubble size, water clarity, and illumination (which may vary with wave phase and bubble density). The distance-induced size error for in-focus bubbles was estimated by moving a grid along the camera axis and noting the distance range and resolution over which they were sharp. Scale variations were $\pm 25 \%$ for the wide FOV camera setting (25-mm lens, $45 \mathrm{~cm}$ to measurement volume) and $\pm 10 \%$ for the narrow FOV camera (50-mm lens). Rather than attempt a direct determination of the measurement volume, the bubble population is defined as the total number of bubbles in the entire plume in a size range. Intercalibration between the mini- and large-BMSs conducted in the a tank at the TNO Physics and Electronics Laboratory (TNO-FEL) for a single aerator also demonstrated good agreement for the overlapping radii ranges, as did observations of the aerators during LUMINY (see section 4).

A comparison of the population for a large (highly turbulent and therefore homogeneous) bubble plume observed by two cameras with different optics showed excellent agreement, accounting for the different optics of the two cameras. An example of a large plume population for two cameras is presented in section 4 .

\section{b. TNO mini-bubble measuring system}

The mini-BMS is a miniaturized version of a larger system previously developed by TNO-FEL for deployment in the open sea (De Leeuw and Cohen 1994). The mini-BMS was designed to measure the bubble size distribution for $15 \mu \mathrm{m}<r<500 \mu \mathrm{m}$ for LUMINY as the large system would have overly perturbed the wave field in the relatively small-scale laboratory, thereby affecting wave processes. Bubbles entered the sample volume by natural advection and were imaged by a video camera. After image processing, the bubble size distribution was calculated.

An overview camera was added to the system for two purposes. First, the overview camera imaged the sample volume and thus provided a check on possible bubble formation or flow modification by interaction with the system's surfaces. Except when the mini-BMS penetrated the air-water interface, this effect appeared to negligibly influence observations. Second, it provided qualitative observations of the sampled bubble plume density. Other improvements of the mini-BMS with respect to the open-sea BMS were a more homogeneous illumination of the sample volume by a continuouswave light-emitting diode (LED) instead of a pulsed laser diode, and a miniature CCD video camera with a built-in electronic shutter. Due to the monochromatic laser light, the pulsed laser diode caused fringes (circular interference patterns) around small particles in the water and on the optics. The built-in electronic shutter of the CCD camera eliminated the need for a pulsed light source.

A schematic drawing of the mini-BMS is shown in Fig. 3. To keep the system compact, the light beam is deflected back to the mini-BMS by two mirrors, $\mathrm{M}_{1}$ and $\mathrm{M}_{2}$, mounted in the corners of tubes that served as light guides. The tubes were equipped with two windows, $\mathrm{W}_{1}$ and $\mathrm{W}_{2}$. The space between $\mathrm{W}_{1}$ and $\mathrm{W}_{2}$ determined the depth of the sample volume, as discussed further below. The diameter of these conical tubes near the windows was $2.0 \mathrm{~cm}$. The tube lengths were chosen so that bubbles pass the light beam only where they are in the focus of the telescope and the bubble size can be determined accurately. The tube ends were shaped conically to reduce turbulence generation near the sample volume. The mini-BMS was approximately $23 \mathrm{~cm} \times 43 \mathrm{~cm} \times 11$ $\mathrm{cm}$, including the conical tubes, weighed $5 \mathrm{~kg}$, and was neutrally buoyant when submerged. The power supply and electronics for the camera and light source were mounted internally on a printed circuit board.

\section{1) Optics AND ILLUMinAtion}

The optical system of the mini-BMS consisted of a light source, a telescope, a CCD detector, plus mirrors 


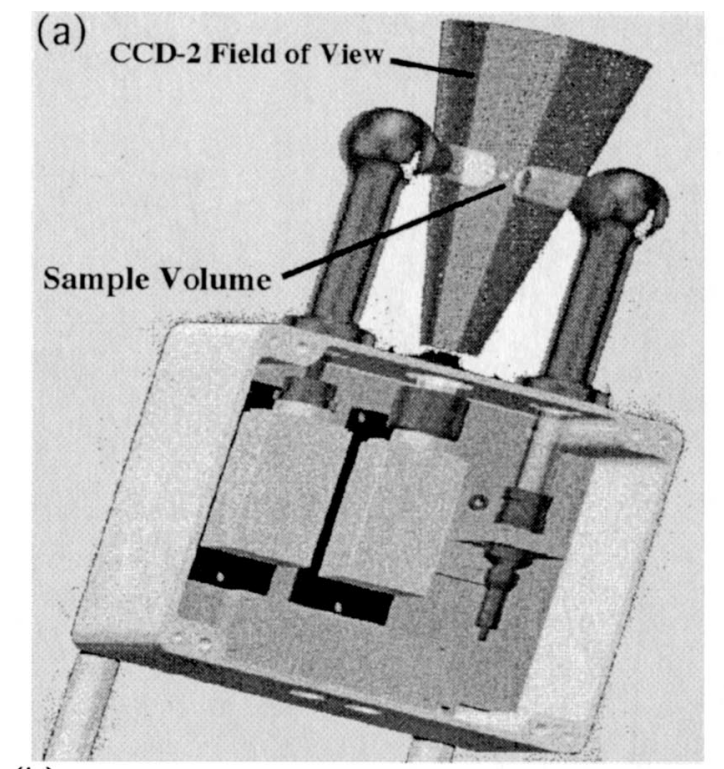

(b)

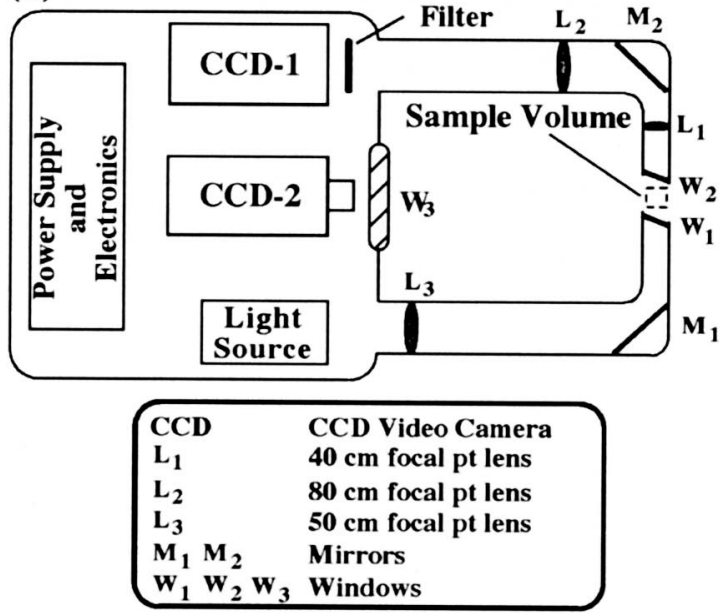

FIG. 3. Artists conception of the TNO-FEL mini-BMS: (a) the measurement volume is determined by the distance between the tubes and the width of the light beam; the measurement volume is inside the conical field of view of the overview camera; (b) block schematic with key.

and windows. The light source was an LED with a wavelength of $880 \mathrm{~nm}$ and a power of $50 \mathrm{~W} \mathrm{sr}^{-1}$. The angular width of the light beam was $15^{\circ}$. The LED was placed in the focal plane of a lens $(f=50 \mathrm{~mm})$ to create a parallel light beam and homogeneous illumination of the sample volume. The sample volume was monitored by a CCD camera (CCD-1), equipped with a daylightblocking filter $(780 \mathrm{~nm})$ and a telescope consisting of two lenses, $L_{1}$ and $L_{2}$, with focal lengths of 40 and 80 $\mathrm{mm}$, respectively. The telescope provided an enlargement factor of 2. The FOV for CCD-1 was $3.15 \mathrm{~mm} \times$ $2.35 \mathrm{~mm}$ with a resolution of $752 \times 582$ pixels.

Because an antireflection coating is unsuitable for use under water, uncoated windows were used. To avoid spurious reflections, they were mounted parallel at an angle of $5^{\circ}$ with respect to the optical axis of CCD- 1 . Parallel mounting was required to prevent distortions arising from refraction effects due to the different densities of water (where the bubbles were sampled) and air (inside the tubes). Because the optical paths in water and air are different, the optics must be aligned with the sample volume under water. In the optical setup with parallel windows the light beam was shifted by a few millimeters.

\section{2) SAMPle VOlume}

The sample volume depth was delimited by the windows $\mathrm{W}_{1}$ and $\mathrm{W}_{2}$ at the end of the conical tubes. Furthermore, only bubbles located in the camera FOV were counted. Thus, the sample volume was determined by the camera FOV and the separation between the windows $\mathrm{W}_{1}$ and $\mathrm{W}_{2}$. The use of a boundary box in the processing algorithm further reduced the sample volume. The boundary box is a rectangle, smaller than the camera FOV, limiting the analysis to bubbles that are completely in the FOV, that is, that do not touch the FOV edge. This resulted in a more accurately defined FOV and thus sample volume. The window separation was $2.0 \mathrm{~mm}$ and the sample volume was $20 \mathrm{~mm} \times 2.9$ $\mathrm{mm} \times 1.9 \mathrm{~mm}$, or $110.2 \mathrm{~mm}^{3}$.

\section{3) Calibration}

The mini-BMS was calibrated with simulated bubble targets, that is, circles drawn on paper that were photographically reduced onto plastic. By using several simulated bubble sizes, a calibration curve was obtained. On the basis of this exercise, the smallest bubble that could be measured reliably was $r \sim 15 \mu \mathrm{m}$. Although smaller bubbles were observed, a minimum number of pixels threshold was applied to accept a bubble for analysis. The small-resolution limit was slightly larger than expected due to the rectangular pixels of the CCD camera. Due to the pixel shape, and also because bubbles smaller than $15 \mu \mathrm{m}$ may not be aligned with the pixel orientation, these smallest bubbles were rejected since they did not satisfy the circularity criterion (see below).

\section{Analysis}

Both systems determined bubble size by discriminating the bubble from the background by image thresholding. A series of calibration experiments determined that the appropriate threshold intensity was slightly below the background intensity (Leifer et al. 2003, manuscript submitted to Chem. Eng. Sci., hereafter L03). In-focus bubbles are dark, surrounded by a white ring with a white central spot. The ring was found to be caused by off-axis light rays reflecting off the front of the bubble and is discussed in L03. Slightly out-of-focus bubbles also exhibited the ring, but without the central 
white spot. The large-BMS observed bubbles of a size range significantly larger than other types of particles in the water and thus was able to discriminate even slightly unfocused bubbles, sizing them through trajectory analysis as described below.

The bubble equivalent spherical radius $r$ was calculated by (Sam et al. 1996)

$$
r=\sqrt[3]{a b^{2}}
$$

where $a$ and $b$ are the major and minor axes radii, respectively. The series of bubble sizes were then combined into a bubble distribution by calculation of a histogram. The calculated distributions were normalized to \# $\mu \mathrm{m}^{-1}$ by dividing by the bin width.

The well-defined sample volume of the mini-BMS and the bubble size calibration allowed the calculation of the concentration distribution. An automatic algorithm was developed that determined $r$. Due to the small sample volume, bubbles generally were observed singly, seldom overlapping. And given the uniform illumination, processing of the mini-BMS data was relatively simple. The large-BMS, on the other hand, often required manual processing to distinguish touching or obscured (i.e., overlapping) bubbles. It is a relatively small step to identify bubbles in subsequent images and thus track them in time. This yields unique information on processes taking place in the bubble plume, as well as the evolution of the bubble plume and bubbles inside the plume. The large-BMS also provided time-resolved distributions.

\section{a. Large-BMS analysis}

Automated routines were used for analysis of the bubble video for the bubble aerators and calibration experiments. After acquiring the video, image processing and analysis were performed using NIH Image $(\mathrm{NIH}$ 2001). Noise was removed from the images by a combination of Gaussian smoothing and La Place sharpen filters. Background intensity variations were subtracted and the contrast enhanced. Bubbles were then identified by built-in NIH Image particle-analysis routines and subjected to an eccentricity test. When a bubble's major axis exceeded the minor axis by more than $15 \%$, it was left for manual identification. Bubbles were analyzed for each movie frame, and the time, location, and the major and minor axes from the best-fit ellipse were determined. For each bubble, $r$ was calculated using (1) and the time series was size segregated into selected intervals to generate time-resolved population distributions. A size histogram was calculated for each time interval with either logarithmically or linearly varying bins between the minimum effective resolution and $5000-\mu \mathrm{m}$ radius (the largest observed bubble size). Distributions were normalized to units of inverse-micrometers.

The results from the large-BMS are presented as bubble population distributions, that is, total number of bub- bles per radius unit in the plume. This is in contrast to the more common convention of bubble concentration distribution, that is, the number of bubbles per radius unit per volume unit. While concentration is a meaningful concept for a quantity that is roughly homogeneous, bubble plumes are highly inhomogeneous, with local concentration gradients that may vary by several orders of magnitude, particularly for large bubbles. Hence, presentation of the results as concentrations rather than populations requires spatial and temporal referencing within the plume. However, in trying to estimate the bubble plume contribution to some bubble process-for example, air-water gas transfer-the total number of bubbles in the plume is required (Leifer 1995). Therefore, conversion of plume concentrations to a plume population requires the (time varying) plume volume.

For bubbles within the plume, bubble size was generally determined through manual outlining. Manual analysis in combination with bubble trajectories allowed the measurement of blurry and obscured bubbles. Thanks to differences in sizes, almost all bubbles were identifiable in adjacent frames, and the trajectories calculated. Bubbles that were blurry due to fast motion during injection were sized when they slowed down, while those that were blurry due to being out of focus were sized only if they became in focus. An image sequence illustrating trajectory analysis is shown in Fig. 4. Images are $0.02 \mathrm{~s}$ apart, and the size scale is indicated by the grid, with black arrows for the bubbles' trajectory. The four trajectories for Figs. $4 \mathrm{a}-\mathrm{c}$ have been combined and superimposed as a white path. Although in this case the tracked bubble was not obscured, trajectory analysis was extremely useful in dense bubble plumes.

Trajectory analysis was used to predict the location of bubbles that disappeared behind other bubbles. Typically, once the predicted location of the hidden bubble was determined, its outline could be seen through the obscuring bubble. Although the vast majority of bubble trajectories were identified, when a bubble possessed a large velocity component in the camera line of view (i.e., $y$ direction), or when several bubbles were of similar size, located close together, and moving in a sufficiently turbulent flow, bubble tracking was not possible. Success at determining the bubble trajectories may be partially explained if, with the exception of the intense turbulence observed at the end of the injection phase, velocities were primarily two-dimensional, that is, along the wave orbital motions. Trajectory analysis also provided information on the fluid dynamics of the bubble plumes and their evolution.

\section{AutOMATED ANALYSIS LIMITATIONS}

Automated analysis routines were unable to be applied reliably for most bubble images from the largeBMS due to the BMS design requirements; that is, they were noninvasive to allow observations of plume for- 

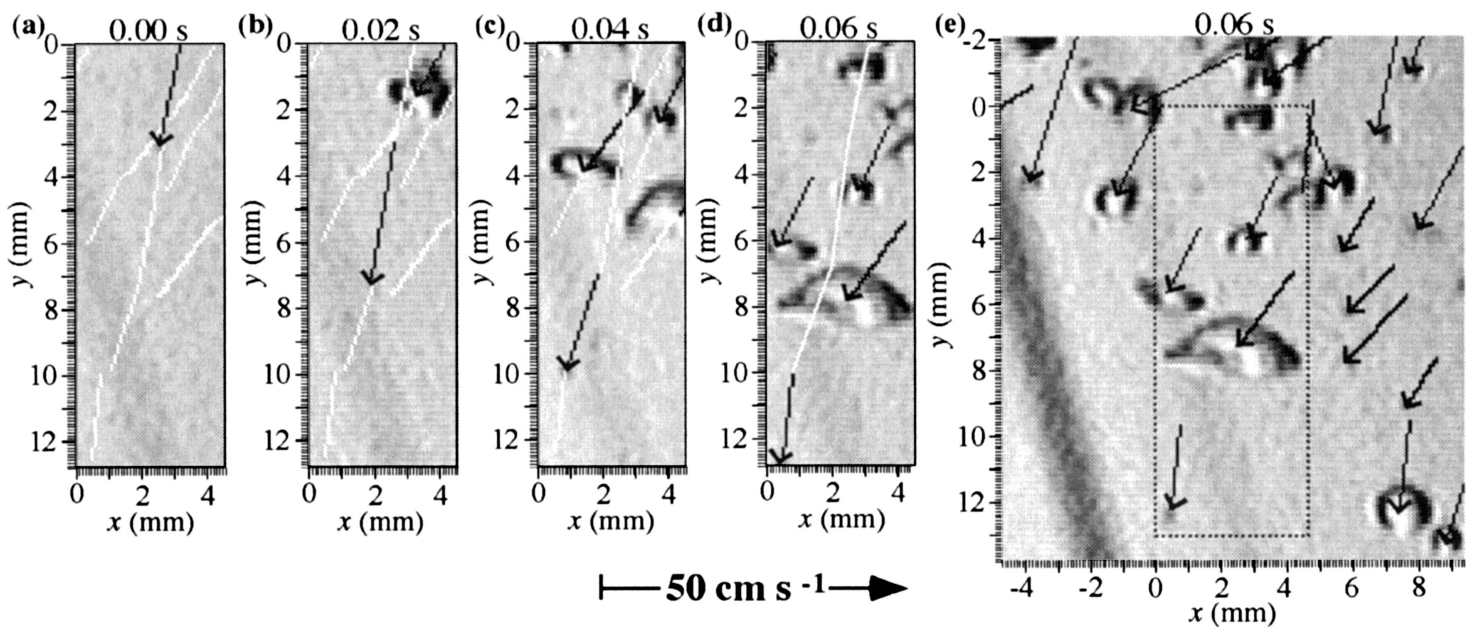

FIG. 4. Time sequence of bubble images with trajectories overlaid. Complete trajectory shown in white, with the trajectory for each image shown in black. (e) Larger view image with dashed rectangle showing location of (a)-(d). Velocity scale shown under panels.

mation, bubbles near the interface, and plume dynamics. This required a large distance between the camera housings and the measurement volume. As a result of the distance, the camera was aimed slightly upward to avoid the interface of the three-dimensional waves from blocking the camera. Due to this tilt, the bright and "textured" interface formed at least part of the background whenever the wave trough was in the FOV. When the interface was significantly above the FOV, the background intensity was very uniform. However, when the interface was close to the FOV, the background intensity varied widely with position and time throughout the image, interfering with automated identification routines.

A second problem was related to the minimum size for bubble identification (i.e., determination that the bubble is in focus). Bubbles smaller than $r \sim 6$ pixels did not have an obvious white central spot. For smaller bubbles, the presence of a white ring surrounding the bubble was used to indicate that the bubble was in focus. This allowed bubbles in the range $300 \mu \mathrm{m}<r<5000$ $\mu \mathrm{m}$ (the largest bubbles observed) to be analyzed while still allowing plume dynamics visualization (requiring an FOV of $\sim 10 \mathrm{~cm}$ ). Unfortunately, the white ring was typically faint (i.e., noisy) and difficult for automated routines to distinguish.

Since bubbles begin to oscillate for $r \sim 700 \mu \mathrm{m}$ (Clift et al. 1978), most of the observed bubbles were susceptible to shape oscillations. For larger bubbles these could be very extreme, particularly during the injection phase when bubbles frequently bounce off each other. Shape irregularities can cause false results for eccentricity and perimeter tests. Automated routines have enormous difficulty discriminating between several large bubbles that appear to be touching and a single, large, deformed bubble. One method uses Houghton transforms, which finds circular shapes in images (NIH Image 2001). Small circular or elliptical bubbles can also be identified by the method of bisecting arcs (Q. Wang 2001, personal communication). However, for large bubbles in the intense turbulence of the bubble plume, shape irregularities cause single bubbles to have multiple bisectors. When multiple irregularly shaped bubbles overlap, automated analysis becomes even more difficult.

In summary, most of the analysis within the bubble plumes was performed manually. Although this limited the number of plumes that could be analyzed, it allowed bubble distributions to be calculated within the bubble plumes.

\section{b. Mini-BMS analysis}

The analysis of the mini-BMS data was developed as an automated algorithm that can be routinely applied to larger datasets. Video signals were both recorded on SVHS video tape and digitized by a dedicated image processing board for continuous analysis of the size and shape of objects in the sample volume. This image processing board was developed and built by TNO and contained a frame grabber and hardware image processing functions such as noise filtering, erosion, etc. After digitization, the pixel size was $6 \mu \mathrm{m} \times 8 \mu \mathrm{m}$. Due to the higher resolution, bubble radius was measured in the horizontal direction.

Because of the sizes covered by the mini-BMS, not only bubbles may be detected but also particles such as suspended matter and biological organisms (e.g., algae). A criterion was applied during data processing to discriminate between spheriods (potential bubbles) and other shapes (nonbubbles). Bubbles in the mini-BMS size range are very close to spheroidal (Clift et al. 1978) whereas other objects may not be. The discrimination criterion uses the aspect ratio $A_{R}$, defined as the ratio between the object's horizontal and vertical dimensions as calculated for each object. However, $A_{R}$ has the dis- 
advantage of being sensitive to object orientation. Thus, a new parameter, circularity $c$, was introduced:

$$
c=\frac{C^{2}}{A}
$$

where $C$ is the object circumference and $A$ is the object area. Manual observations of a large number of samples showed that a value of 17 , or an aspect ratio of 2.3 , was appropriate to distinguish between bubbles and algae.

A second criterion to discriminate between bubbles and other objects was the appearance of bubbles as dark objects with small white central spots, while slightly out-of-focus bubbles were completely dark, as discussed above for the large-BMS. In contrast, most other objects such as algae are partially transparent. In the mini-BMS algorithm, a transparency criterion was set to accept only objects where the ratio of the number of black to white pixels in the object was larger than $90 \%$.

Partially overlapping bubbles were separated by pixel erosion, that is, removing the outer pixel layers of the combined object until the two objects are separate. If the circularity and transparency criteria are satisfied, they are classified as bubbles and counted. For sizing, the eroded pixels were taken into account. The measured radii were then sorted into 20 logarithmically spaced intervals spanning $15 \mu \mathrm{m}<r<500 \mu \mathrm{m}$ with increasing width. The number of bubbles in each size bin was counted and then divided by the product of sample volume, sample time, and bin width, to calculate the bubble size distribution $d \Phi / d r\left(\# \mathrm{~cm}^{-3} \mu \mathrm{m}^{-1}\right)$. To obtain statistically meaningful samples, the sampling time was generally $15 \mathrm{~min}$, although it was varied according to wave conditions.

\section{Aerator bubble observations}

Bubble gas transfer during LUMINY was due to bubbles produced by breaking waves, aerator beds, or a combination of both. The purpose of the bubble aerators was to achieve a controlled simulation of the effect of bubbles without wave breaking and the associated turbulence and wind stress on gas transfer and is described in more detail in De Leeuw et al. (1999). The aerators' air source was either external to the wind tunnel or from the tunnel headspace gas (i.e., in contact with the water surface and thus directly involved in the air-water gas transfer process). In addition, the aeration flow rate could be varied. The large- and mini-BMSs were mounted on a movable chariot that scanned the tunnel length, traversing the aerator beds. Aerators were randomly located on the aerator beds and were at a fixed depth below the water surface (despite the slight slope of the tunnel floor).

The aerators produced one of two distinct bubble distributions, either a narrow, sharply peaked distribution or a broad distribution of large bubbles. Images and populations from bubble streams generated by the two aerator types are shown in Fig. 5. The distribution from
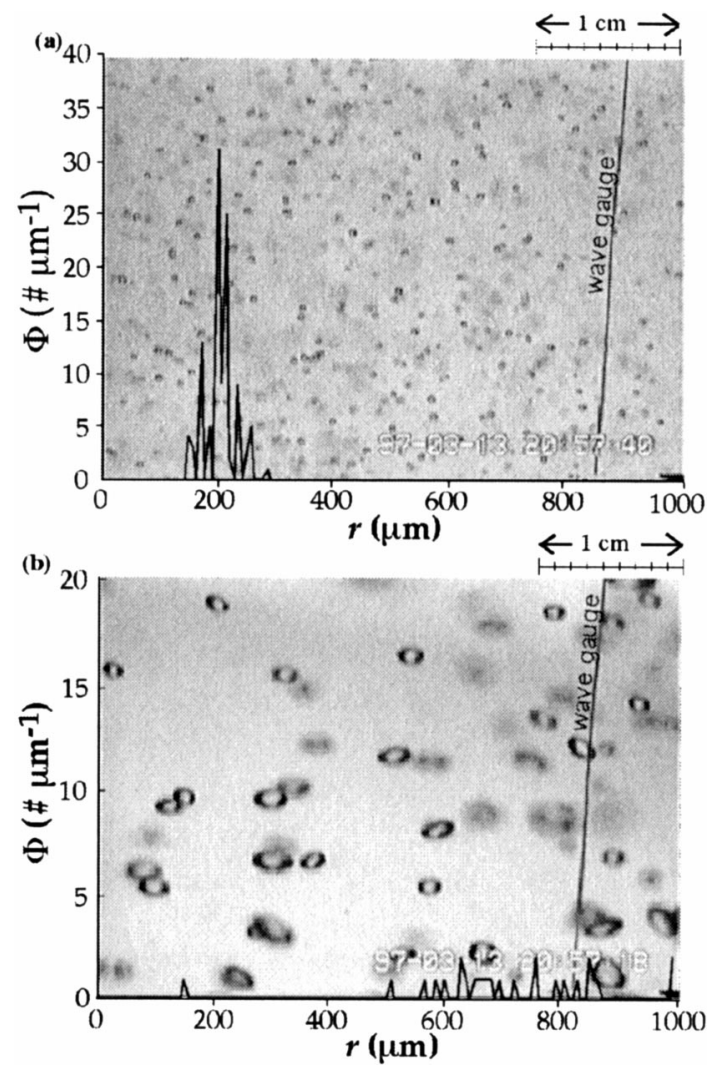

FIG. 5. Sample images from the two aerator types and the calculated population distributions $\Phi$ for the images shown. The vertical line is the wave gauge. Length scale is shown to upper right of each image; smaller ticks are $1 \mathrm{~mm}$.

the aerator shown in Fig. 5a was very narrow, with a peak near $r=205 \mu \mathrm{m}$, and a half-width of $\sim 20 \mu \mathrm{m}$ determined by where $\Phi$ decreased from the peak concentration by $e^{-1}$. The half-width probably resulted from a combination of 1) physical factors, such as aerator pore size and shape, bubble shape oscillations; and 2) observational factors, such as distance from the camera, which causes changes in apparent size. Although similar-sized bubbles in stagnant fluid do not oscillate (Leifer et al. 2000c), shape oscillations occurred for these bubbles, probably due to bubble-bubble (Duineveld 1998) and bubble-wake interactions (Fan and Tsuchiya 1990). Bubbles from the second aerator type (Fig. 5b) were significantly larger and the distribution was very broad (half-width $\sim 250 \mu \mathrm{m}$ ) with a peak at $r \sim 750$ $\mu \mathrm{m}$. While bubbles this large normally oscillate, the distribution broadness is significantly larger than for individual bubbles of a similar size (Leifer et al. 2000c). Thus, the broad half-width of the second population (Fig. 5b) must be due to variability in bubble formation.

The global aerator bubble population is shown in Fig. 6 for both the large- and mini-BMSs. The distributions were combined by normalizing the large-BMS to the mini-BMS concentration for the $275-\mu \mathrm{m}$ peak. The normalization accounts for the different measurement vol- 


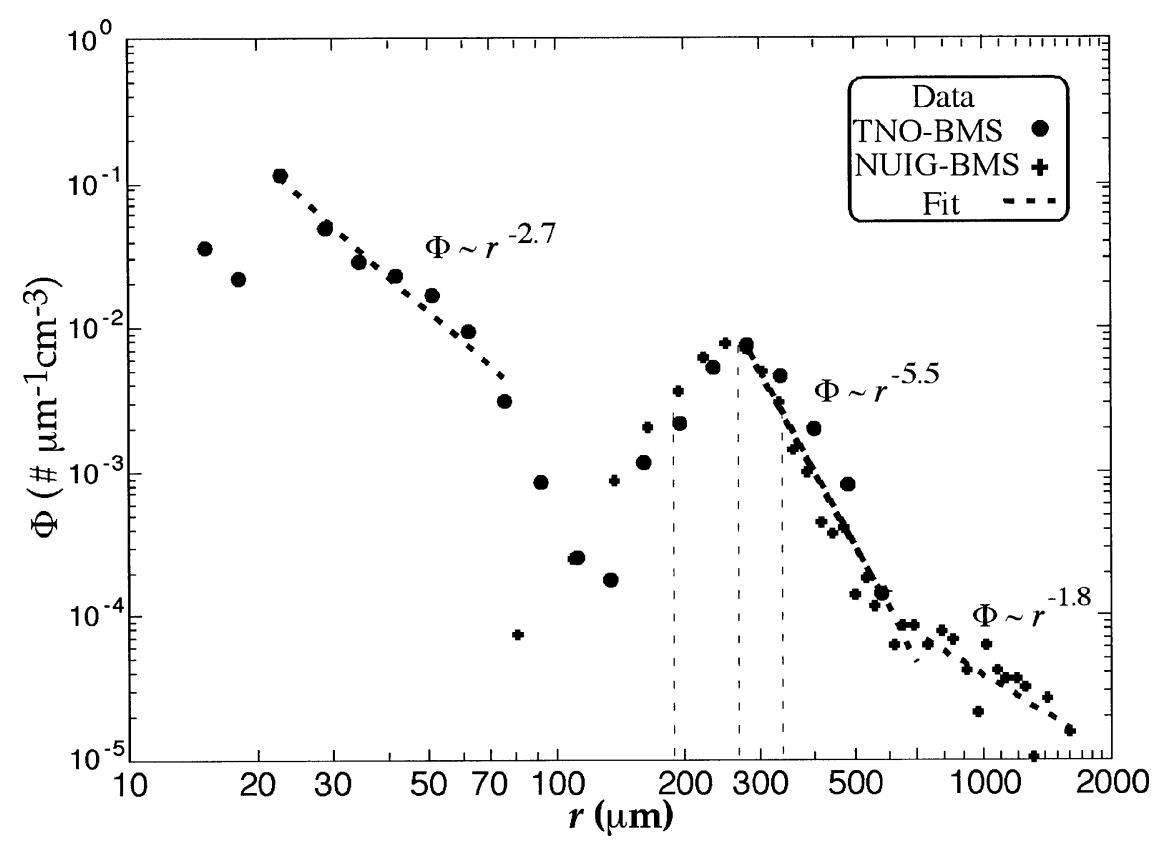

FIG. 6. Normalized, global bubble population distributions $\Phi$ as function of radius $r$ and power-law distribution fits for bubble aerators. Data key on figure.

umes. The power-law dependency of the bubble distribution is given by

$$
\Phi(r)=k r^{-S(t)},
$$

where $\Phi$ is the population distribution, $S$ is the power law and may vary with time $t$, and $k$ is a constant. Here $S$ was determined by a least squares, linear-regression analysis to the logarithm of both sides of (3). The powerlaw fits are also shown in Fig. 6. Agreement between the two datasets is very good for $150 \mu \mathrm{m}<r<500$ $\mu \mathrm{m}$, that is, the common size range. If the efficiency varied differently with $r$ for the two BMSs, $S$ would be different and the peaks would not align. The distribution shows a narrow peak centered at $275 \mu \mathrm{m}$ that decreased very steeply with $r(S=-5.5)$. The peak half-width was $70 \pm 20 \mu \mathrm{m}$. The decrease in $S$ to -1.8 for $r>$ $600 \mu \mathrm{m}$ is due to the contribution from the large bubbles from second aerator type (Fig. 5b). The mini-BMS observed a third bubble population with a peak at the small-resolution limit. This population was shallower, with $S=-2.7$. One possible explanation for this very small bubble population may be bubble-burst bubble production. Specifically, when bubbles burst, the descending bubble film can produce many small bubbles. The value of $S$ and the size range of this bubble population are consistent with laboratory observations of bubble-burst bubble production (Leifer et al. 2000b).

\section{Bubble blurring and measured bubble size}

Given sufficient illumination, current video cameras allow selection of shutter speeds sufficiently fast to prevent bubble blurring. However, if illumination is lim- ited, a choice must be made between aperture size (i.e., DOF) and shutter speed (blurring). The decision depends upon the bubble distribution and bubble dynamics of interest. For example, in a constrained system, the DOF need only be the distance between windows, while a large DOF may be required in an unconstrained system with the goal of visualization of bubble plumes. The effect of bubble blurring depends upon the bubbles' velocity (pixels ${ }^{-1}$ ) and size (pixels). While it is easy to design a BMS with sufficient illumination in the laboratory, at sea [shipboard or submersible/remotely operated vehicle (ROV), etc.] power may be a limiting factor. Furthermore, the illumination required depends upon the size of the FOV. For example, a small FOV is required to study small bubbles; thus, light easily can be focused to evenly and intensely illuminate the small FOV. In contrast, a large FOV requires significantly more illumination power.

Blurring smears the bubble image, but the result may be complex. For blurring of $\sim 1$ pixel or less, blurring can increase sharpness. Consider a bubble whose edge is half in 2 pixels. Smearing the bubble in one direction can place more of the edge in 1 pixel, creating a sharper edge. Alternatively, if the edge is almost entirely in 1 pixel, the same blurring spreads the intensity across 2 pixels, decreasing sharpness.

Also, since blurring occurs along only one direction, blurring may affect only one axis. An example is shown in Figs. 7a,d for a $670-\mu \mathrm{m}$ bubble. These two images were taken during a calibration experiment described below for shutter speeds of $1 / 20000$ and $1 / 500 \mathrm{~s}$, respectively. Furthermore, given that bubble motion is on 
(a)

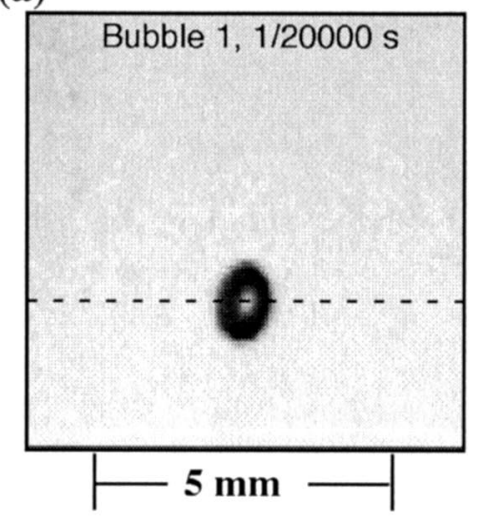

(d)

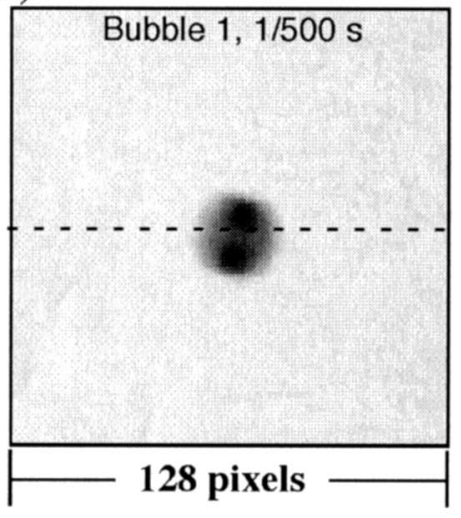

(b)

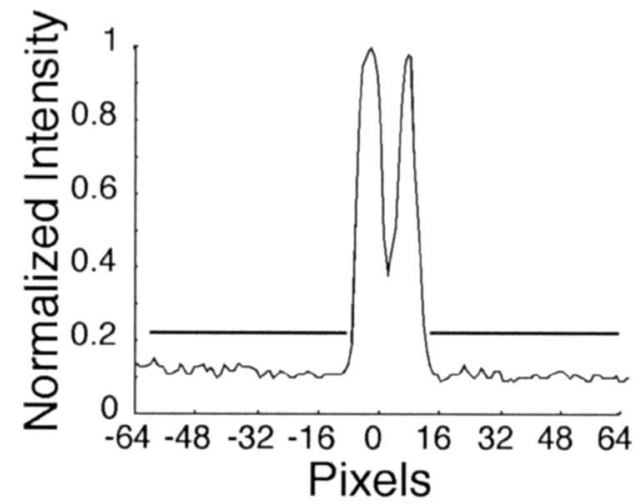

(e)

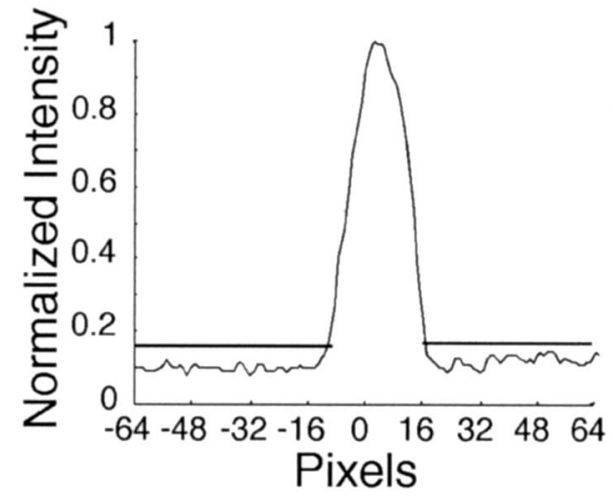

(c)

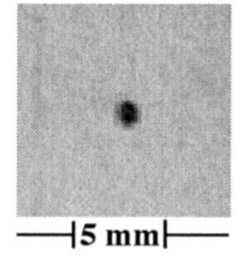

(f)

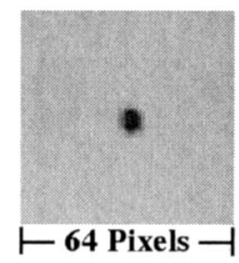

FIG. 7. Images of $670-\mu \mathrm{m}$ bubbles for (a) $1 / 20000$ - and (d) $1 / 500$-s shutter speeds. (b), (e) Intensity profiles for (a) and (d), respectively, for dashed lines in (a), (d). Also shown are 670- $\mu \mathrm{m}$ bubbles at lower resolution for (c) 1/10 000- and (f) $1 /$ 500 -s shutter speeds. Size scales located under images.

average vertical (due to buoyancy), blurring occurs mostly on the minor rather than the major axis [Eq. (1)]. Further complexities arise in the relationship between blurring and apparent size since nonblurred bubbles exhibit intensity gradients at their edges (see Figs. 7b,e) and bubble size is determined by thresholding. Finally, to determine an acceptable level of blurring, blurringinduced error should be compared with other errors and natural variations.

To understand the effect of bubble blurring upon apparent bubble size, a series of bubble blur calibration experiments was conducted in a glass tank and tap water $\left(20^{\circ} \mathrm{C}\right)$ at the University of California, Santa Barbara. A schematic is shown in Fig. 8. A regulated airflow was fed through one of two drawn capillary tubes to generate single streams of bubbles (Leifer et al. 2000c). Bubbles were imaged with a high-speed video camera (Kodak, Ektapro, 1000 FPS) for shutter speeds ranging from $1 / 60$ to $1 / 20000 \mathrm{~s}$. Backlighting was provided by a $1000-\mathrm{W}$ spotlight with $2^{\circ}$ dispersion illuminating a translucent plastic screen located behind the tank. Distances between the camera backplane, bubbles, and screen are provided in Table 1, as well as other parameters. Illumination was controlled by a var- iac and varied to allow consistent background intensity for an aperture of f8, except at the fastest shutter speeds $(\leq 1 / 10000 \mathrm{~s})$ where the aperture was f5.6. A 55-mm telecentric lens (Computar, Japan) was used, thereby minimizing errors due to uncertainty in the distance to the bubble. From the highest shutter speeds, the two capillaries produced $r=670$ - and 2240- $\mu \mathrm{m}$ bubbles. Bubble sizes were consistent during each run.

Image sequences were digitized at a resolution of 720 pixels $\times 480$ pixels, cubic-spline interpolated to double resolution and thresholded 10 intensity units above the local background (determined by a 10-pixel-wide ring around each bubble). Major and minor axes (and angle) were determined from a least squares linear-regression fit of an ellipse to the bubble outline.

Bubble size distributions for $670-\mu \mathrm{m}$ bubbles (experiment 1, Table 1) for 1/20 000- and 1/500-s shutter speeds (e.g., Figs. 7a,d) are shown in Figs. 9a,b, respectively. Also shown in Figs. 9c,d are size distributions for larger, 2240- $\mu \mathrm{m}$ bubbles (experiment 3, Table 1) for $1 / 10000$ and $1 / 250 \mathrm{~s}$, respectively. Each distribution represents several thousand bubble images. The motion of the $670-\mu \mathrm{m}$ bubbles at $1 / 20000 \mathrm{~s}$ was 0.2 pixels, while for $1 / 500 \mathrm{~s}$ it was 12.4 pixels. Since the 


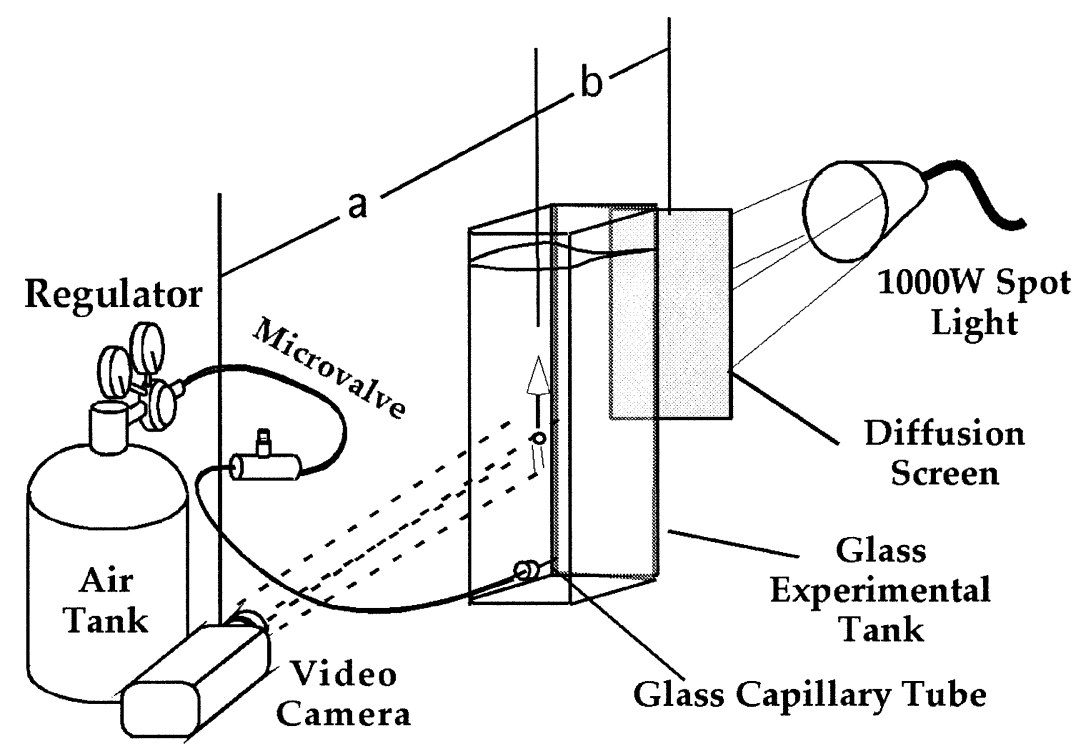

FIG. 8. Schematic of bubble blur calibration experiment.

bubble diameters were $\sim 24$ pixels, the $18 \%$ size increase (from 670 to $790 \mu \mathrm{m}$ ) is less than might be expected for blurring comparable to $r$. The primary reason is that although the bubble is spread over more pixels, the intensity of those at the leading and trailing edges is greatly reduced, and thus the threshold level is above the broadened intensity. Also contributing is the fact that only the minor axis is blurred. For the larger and slower $2240-\mu \mathrm{m}$ bubbles ( 12 pixels s ${ }^{-1}$ for $1 / 250$-s shutter speed), the effect of blurring is even less. Also note that oscillations broadened the size distribution for large bubbles increasing the uncertainty in $r$.

The measured bubble $r$ versus shutter speeds are shown in Fig. 10 for all three runs. Errors bars are one standard deviation. For $670-\mu \mathrm{m}$ bubbles and the narrow FOV (run 1), blurring had negligible effect for shutter speeds greater than $1 / 1000 \mathrm{~s}(6.2$ pixels blur, $\sim 50 \%$ of $r$ ), At slower shutter speeds, the error grew rapidly. For the same bubbles but with a wide FOV (run 2) bubbles were $\sim 5$ pixels in radius and thus sizing errors are larger. In fact, the size overestimate in run 2 was $\sim 1$ pixel. The lower resolution decreases the effect of blurring with increasing shutter speed, only becoming significant for speeds of $1 / 250 \mathrm{~s}$, where the blur was $\sim 7$ pixels (140\%). For oscillating bubbles several effects combine to make bubble blurring less significant. The greater size

TABLE 1. Bubble blur calibration parameters and results. Experimental dimensions $a, b$, and $c$ are shown in Fig. 8; here $V$ is velocity and $r$ is effective spherical radius.

\begin{tabular}{cccccc}
\hline \hline $\begin{array}{c}\text { Expt } \\
\text { run }\end{array}$ & $\begin{array}{c}a \\
(\mathrm{~cm})\end{array}$ & $\begin{array}{c}b \\
(\mathrm{~cm})\end{array}$ & $\begin{array}{c}V \\
\left(\mathrm{~cm} \mathrm{~s}^{-1}\right)\end{array}$ & $\begin{array}{c}r \\
(\mu \mathrm{m})\end{array}$ & $\begin{array}{c}r \\
(\text { pixels })\end{array}$ \\
\hline 1 & 58.7 & 50.9 & 33.8 & 670 & 12.1 \\
2 & 163 & 50.9 & 33.8 & 670 & 4.7 \\
3 & 58.7 & 50.9 & 26.1 & 2240 & 25.4 \\
\hline
\end{tabular}

(in pixels), slower velocity, and greater eccentricity decrease the error due to blurring, particularly in respect to the greater uncertainty in bubble size due to oscillations. To summarize, bubble blurring of less than a pixel was always negligible; however, a larger level of bubble blurring is often tolerable, particularly for bubbles large enough to show a white central spot $(\sim 10$ pixels diameter). For bubbles smaller than 10 pixels,
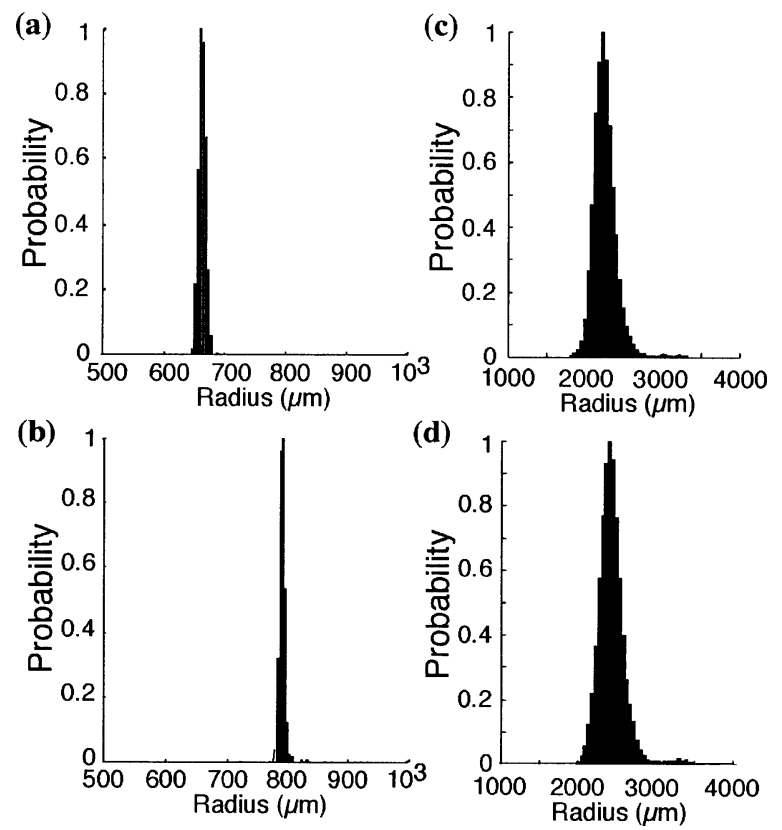

FIG. 9. Bubble size probability distributions for (run 1) $670-\mu \mathrm{m}$ bubbles at (a) 1/20 000- and (b) 1/500-s shutter speeds; and (run 2) $2240-\mu \mathrm{m}$ bubbles at (c) $1 / 10000-$ and (d) $1 / 250$-s shutter speeds. Experimental details in Table 1. 


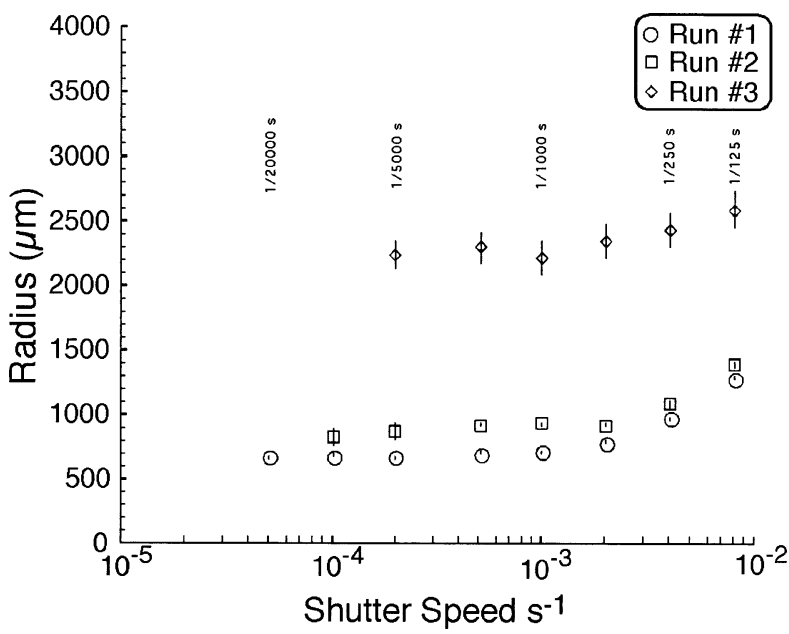

FIG. 10. Bubble radius vs shutter speed for all three experimental runs. Vertical lines are one standard deviation. Run details in Table 1. Data key on figure.

bubble blurring is generally less important, relative to underestimation due to pixilation.

Not addressed in this discussion is the effect of illumination on bubble visualization. A greater intensity is required to visualize blurred bubbles; thus, rapidly moving, small bubbles may fail to be identified. Practical experience showed that this is only a problem for the smallest bubbles (i.e., a few pixels in radius) because larger bubbles exhibit overlapping; that is, a 1-pixel bubble blurred 10 pixels has its intensity everywhere diminished to $10 \%$, whereas a 10 -pixel bubble blurred the same 10 pixels has its intensity diminished $50 \%$ over its central 10 pixels (although more over the first and last 5 pixels.

\section{Bubble observations}

\section{a. Background bubble observations}

The mini-BMS was used to quantify bubble distributions in the background population during LUMINY. Bubble distributions for $13 \mathrm{~m} \mathrm{~s}^{-1}$ wind speed and paddle waves averaged over all fetches are shown in Fig. 11. The concentration decreased steeply with $S=-3.1$. A comparison with laser-based measurements in the same wind tunnel about a decade earlier by Baldy and Bourguel (1987) is also shown in Fig. 11. The Baldy and Bourguel measurements were at a similar depth (8.7 $\mathrm{cm}), 24-\mathrm{m}$ fetch, with a wind speed of $14 \mathrm{~m} \mathrm{~s}^{-1}$ and a paddle frequency of $1 \mathrm{~Hz}$. The Baldy and Bourguel (1987) data show a very similar trend over most radii, with $S=-3.0$. The laser observed larger $(r>30 \mu \mathrm{m})$ bubbles than the mini-BMS. The difference in magnitude between the two is explained by the higher wind speed in the experiments by Baldy and Bourguel (1987) - $14 \mathrm{~m} \mathrm{~s}^{-1}$ as opposed to $13 \mathrm{~m} \mathrm{~s}^{-1}$ during the LUMINY experiments-since Baldy and Bourguel (1987) found a very strong dependency between bubble

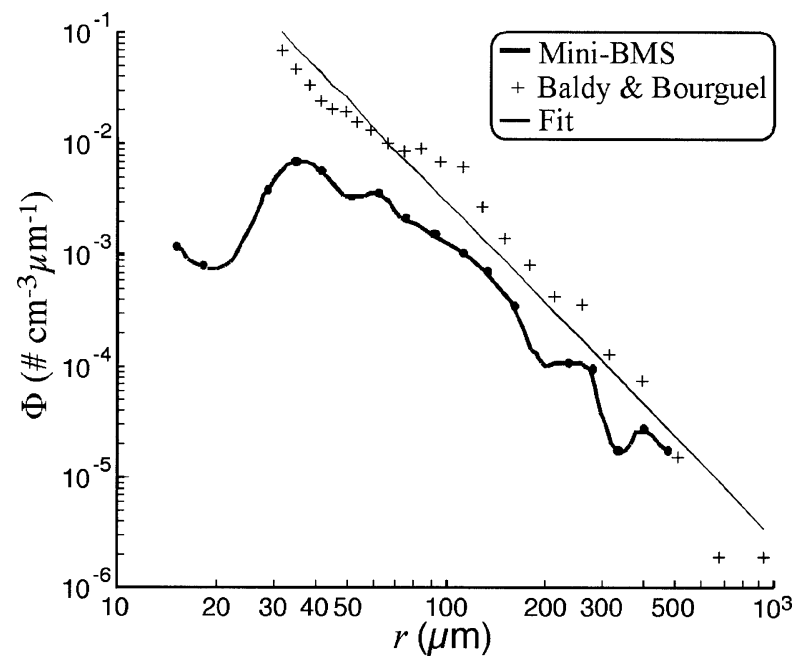

FIG. 11. Bubble concentration size distribution $\Phi$ vs radius $r$ and power-law fit for the LUMINY background population for the miniBMS and Baldy and Bourguel (1987). Fits shown for $r$ range used. Data key on figure.

concentration and wind speed. Another difference between the two observations was the observation fetch. Baldy and Bourguel (1987) measured at 24-m fetch, the region of peak wave breaking during LUMINY (De Leeuw and Leifer 2001), while the mini-BMS observations were averaged over fetches from 17 to $30 \mathrm{~m}$.

\section{b. Bubble plume observations}

The large-BMS was designed to observe noninvasively the dense bubble plumes resulting from wind stress-steepened breaking waves and to provide timeresolved bubble distributions. An example of a timeresolved bubble distribution observed during LUMINY is shown in Fig. 12b for the plume shown in Fig. 12a. The image was during the injection phase of the bubble plume. During injection, the distribution peaked at $r \sim$ $500 \mu \mathrm{m}$ and decreased with $S=-3.7$. Since bubble rise speed increases with $r$, the distribution steepened to $S=-5.4$ by the end of the plume lifetime. Overview camera images of a large bubble plume are shown in Fig. 13. The images were for $t=0.30 \mathrm{~s}$ (injection) and $t=0.86 \mathrm{~s}$ (maximum penetration), where $t$ is relative to the plume formation. Also shown are white rectangles indicating the approximate FOVs of the large and narrow FOV cameras, both of which had their optical axes at $\sim 30^{\circ}$ (to the left) from the overview camera's optical axis. The overview camera was angled slightly upward to include the water surface and also the wave crests.

Images from the wide and narrow FOV cameras for the same time as the overview image shown in Fig. 13a are shown in Fig. 14. A few of the bubbles in Fig. 14a are blurred due to their speed (e.g., $x=1.8 \mathrm{~cm}, z=$ $-1.7 \mathrm{~cm}$, where $x$ is horizontal position and $z$ is vertical position in the image). Other bubbles are blurred due to being too distant or near. For example, the clump of 

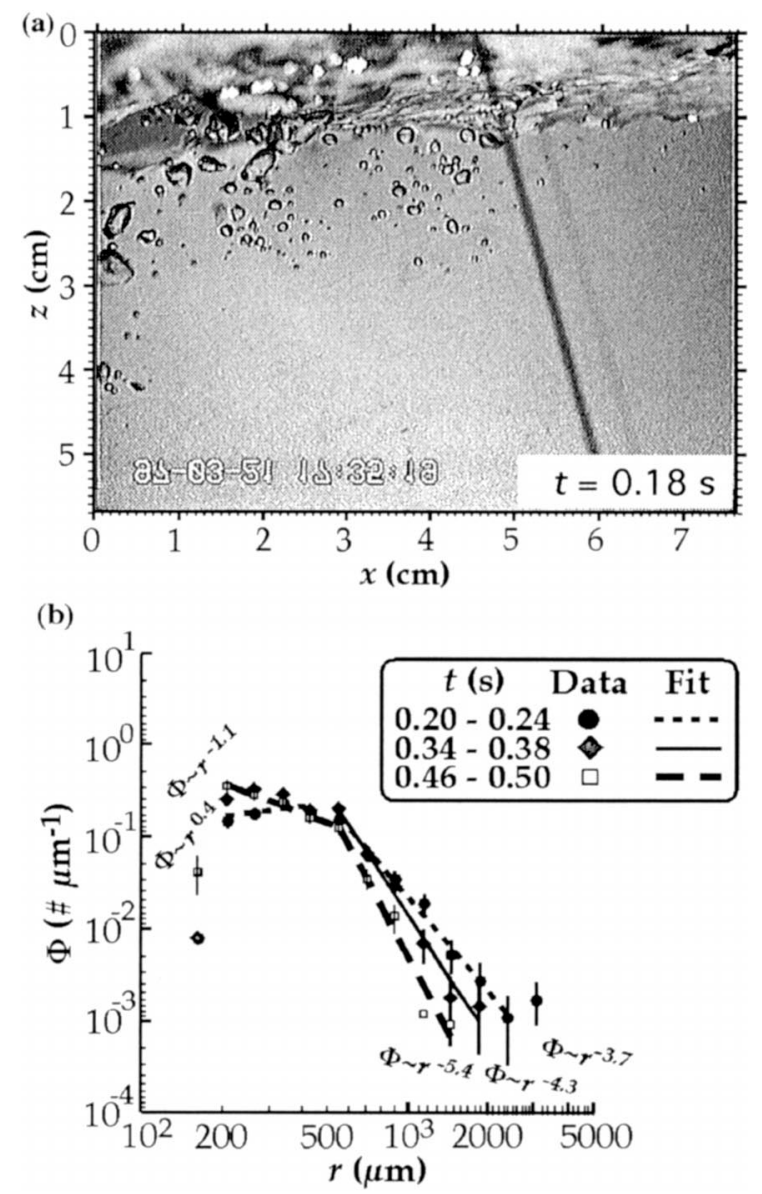

FIG. 12. Image of a shallow plume during the injection phase $(t=$ $0.18 \mathrm{~s}$, where $t$ is time), and the bubble population distribution $\Phi$ as a function of radius $r$ and $t$.

blurry bubbles at $x=4.7 \mathrm{~cm}, z=2.1 \mathrm{~cm}$, was from a second plume in the background visible in the overview camera image (Fig. 14a) to the right of the descending plume. While the DOF was sufficient for the entire plume to be in focus in the wide FOV camera, for the narrow FOV camera (Fig. 14b), it was not. Some bubbles were not counted-for example at $x=0.6 \mathrm{~cm}, z$ $=2 \mathrm{~cm}$-while bubbles in the upper right were. Also note that it is possible to see through bubbles, for example, the two bubbles at $x=1.5 \mathrm{~cm}, z=1 \mathrm{~cm}$, where the outline of the partially obscured bubble is visible through the closer bubble.

The entire image sequence for this plume was analyzed, and the bubble population was calculated for both the wide and narrow FOV cameras. The time-averaged population distribution for the plume is shown in Fig. 15. Each population was scaled by the ratio of the total plume area to FOV area, as observed in the overview camera (see Fig. 13a). The narrow FOV camera $\Phi$ was further scaled by a factor of 2 to account for its narrower DOF (as determined by the ratio of DOFs for the two cameras) due to its longer focal lens.
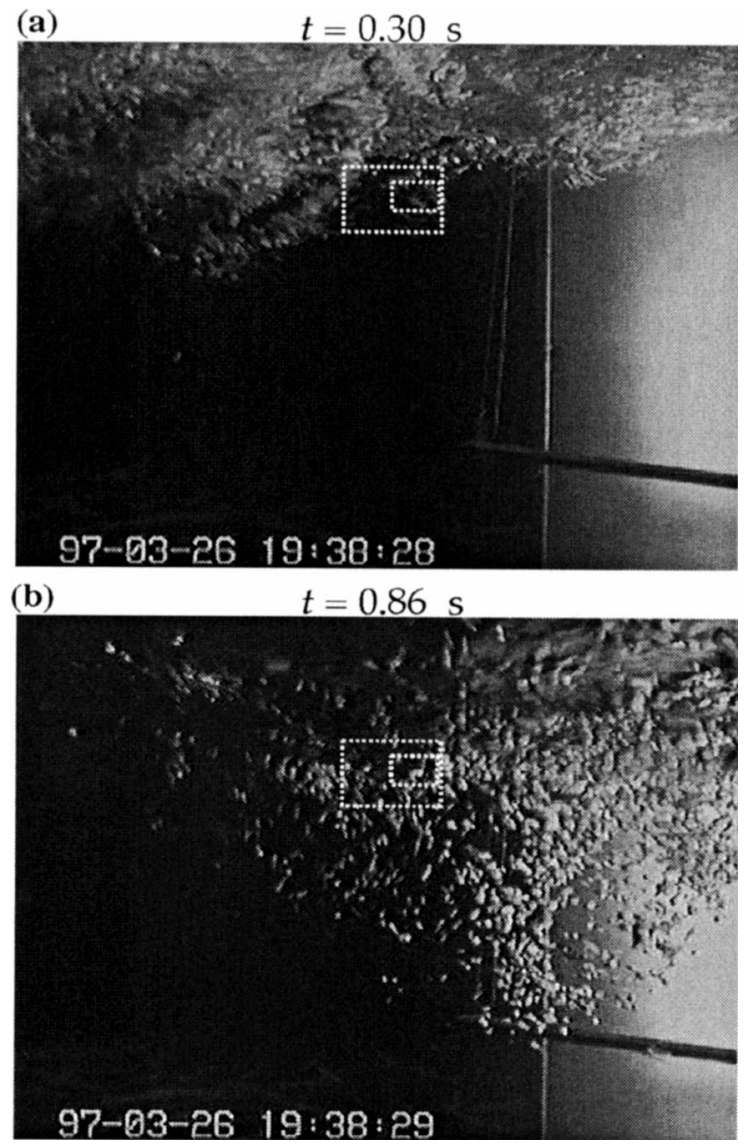

FIG. 13. Images of a large bubble plume from the overview camera at (a) $t=0.30 \mathrm{~s}$ and (b) $t=0.86 \mathrm{~s}$, where $t$ is time after formation. Part of a wave gauge is visible in the image; its lower spar is at $\sim 30$ $\mathrm{cm}$ depth. Dotted white rectangles are approximate location of the wide and narrow FOV cameras.

The agreement between the two distributions over a wide $r$ range demonstrates that, except for the smallest bubbles, the observational efficiencies were close to unity. The effective minimum resolution for the wide FOV is indicated by the divergence of the two curves for small $r$, below which the distribution must be considered unreliable. The effect of the small $r$ resolution limit was significantly larger than theoretical, with the effective minimum $r$ indicated by the divergence in the distributions. For the wide FOV camera the effective minimum size was $r \sim 180 \mu \mathrm{m}$.

This $\Phi$ shows that the plume contained two distinct bubble populations: one large and one small. Most bubble plume distributions observed during LUMINY were either bi- or trimodal. Also shown in Fig. 15 are least squares, linear-regression analysis fits to $\Phi$ for both peaks. Given the homogeneous (i.e., well mixed) nature of this plume, the time-averaged distribution shown is not markedly different from the observed initial distribution. In this regard, the small bubble slope, $S=-2.3$, is in good agreement with bubble observations near the formation region, for example, Baldy (1988). Further 

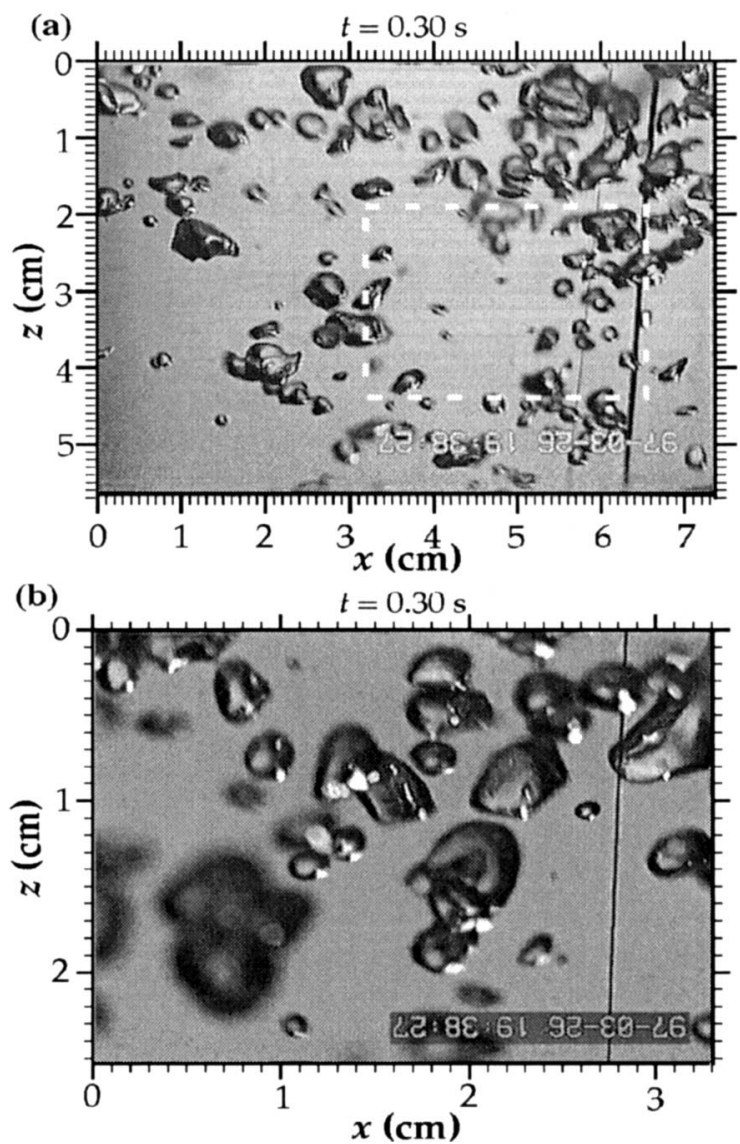

FIG. 14. Images from the (a) wide FOV and (b) narrow FOV cameras at $t=0.30 \mathrm{~s}$ for the plume shown in Fig. 13. White dashed rectangle shows location of narrow FOV. Horizontal $x$ and vertical $z$ scales on figures.

discussion of the BMS bubble observations from the LUMINY experiment are presented in detail in Leifer and De Leeuw (2001).

\section{Conclusions}

In the design of a bubble measurement system (BMS), consideration of the bubble size range of interest is very important. For observing small bubbles, constraining the sample volume, as in the mini-BMS, offers a clearly defined measurement volume without flow disturbances influencing the bubble distribution. In contrast, for observing larger bubbles, or close to the interface, or plume dynamics, a far less invasive system, such as the large$\mathrm{BMS}$, is required. The primary disadvantage of such systems is the poorly defined measurement volume. Many other considerations are important in BMS design such as electrical noise in the environment, proper cable choice, and illumination. For illumination, uniformity and sufficient brightness to allow a high shutter speed and a reasonable depth of field are the most important criteria. Due to the problem of bubble motion blurring, shutter speed selection is an important consideration. De-

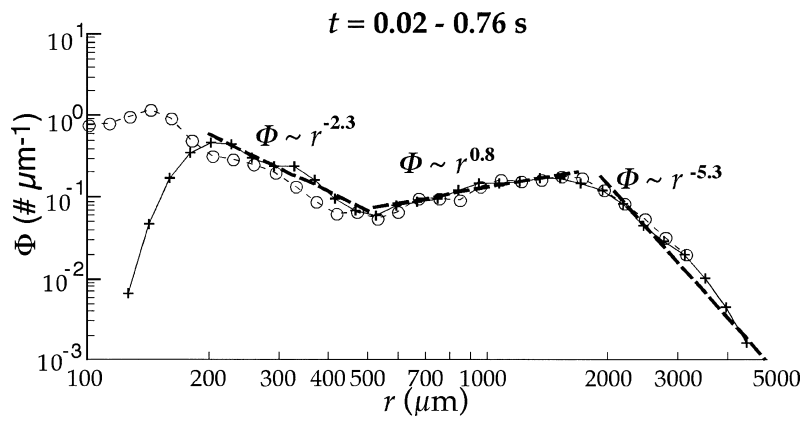

FIG. 15. Time $t$ averaged bubble population distribution $\Phi$ vs radius $r$ for the plume shown in Fig. 14 by the wide FOV camera $(+)$ and narrow FOV camera (O). Dashed line shows fit to wide FOV camera over fit range.

pending upon the bubble size range of interest, different levels of bubble blurring may (or may not) be acceptable.

Under conditions of very high void fraction (e.g., in a bubble plume), automated bubble identification is highly problematic. New analysis approaches will be required to analyze a statistically large number of plumes. Both BMSs described in this paper can and have been deployed at sea. For oceanic deployment, the BMS must be suspended from two buoys that are oriented by means of a bridle attached to a sea anchor in such fashion that the BMS optical axes are themselves aligned perpendicular to the wave direction. This allows the measurement volume to be accessible to bubbles moving in response to wave fluid motions.

The two BMSs were deployed during the LUMINY wind-wave experiment. Intercalibration of the two systems for bubble streams produced by bubble aerators provided very good agreement. Due to the different design criteria of the two systems, they were highly complementary, allowing a combined measurement over a very wide range of bubble sizes, $15 \mu \mathrm{m}<r<5000$ $\mu \mathrm{m}$. The overview cameras provided additional information on plume structure and the context for the observed bubble distributions.

Acknowledgments. Most of the work reported here was carried out as part of the LUMINY project, which was supported by the European Commission EC DG XII, Contract ENV4-CT95-0080, and for the part of TNO-FEL, with additional support from the Netherlands Ministry of Defense, Assignment A95KM786. Part of the work of Dr. Leifer was carried out while he was employed by the National University of Ireland in Galway, and when he was a visiting scientist at TNO-FEL. The bubble blurring calibration was conducted at the University of California, Santa Barbara, and was supported by the U.S. Mineral Management Service. The authors also would like to thank IRPHE-IOA for the extensive laboratory support provided during LUMINY. 


\section{REFERENCES}

Asher, W. E., and P. J. Farley, 1995: Phase-Doppler anemometer measurement of bubble concentrations in laboratory-simulated breaking waves. J. Geophys. Res., 100 (C4), 7045-7056.

— L. L. Karle, B. J. Higgins, P. J. Farley, I. S. Leifer, and E. C. Monahan, 1996: The influence of bubble plumes on air-seawater gas transfer velocities. J. Geophys. Res., 101 (C5), $12027-$ 12041.

- — $—$, and — 1997: On the difference between bubble-mediated air-water transfer in freshwater and seawater. J. Mar. Res., 55, 1-34.

Asselin, C., Y. Comeau, and Q. A. Ton-That, 1998: Alpha correction factors for static aerators and fine bubble diffusers used in municipal facultative aerated lagoons. Water Sci. Technol., 38, 7985.

Baldy, S., 1988: Bubbles in the close vicinity of breaking waves: Statistical characteristics of the generation and dispersion mechanism. J. Geophys. Res., 93 (C7), 8239-8248.

wave crest levels. J. Geophys. Res., 92 (C1), 2919-2929.

Blanchard, D. C., 1963: Electrification of the atmosphere by particles from bubbles in the sea. Progress in Oceanography, Vol. 1, Pergamon, 73-197.

—_ 1989: The ejection of drops from the sea and their enrichment with bacteria and other materials: A review. Estuaries, 12, 127137.

Bowyer, P., 1992: The rise of bubbles in a glass tube and the spectrum of bubbles produced by a splash. J. Mar. Res., 50, 521-543.

Cipriano, R. J., and D. C. Blanchard, 1981: Bubble and aerosol spectra produced by a laboratory breaking wave. J. Geophys. Res., 86 (C9), 8085-8092.

Clift, R., J. R. Grace, and M. E. Weber, 1978: Bubbles, Drops, and Particles. Academic Press, 380 pp.

Deane, G. B., and M. D. Stokes, 1999: Air entrainment processes and bubble size distributions in the surf zone. J. Phys. Oceanogr., 29, 1393-1403.

De Leeuw, G., 1990: Spray droplet source function: From laboratory to open ocean. Modeling the fate and influence of marine spray. Proceedings of a Workshop Held 6-8 June 1990, Luminy, Marseille, France, P. G. Mestayer et al., Eds., Marine Science Institute, University of Connecticut, 17-28.

- and L. H. Cohen, 1994: Measurements of oceanic bubble size distributions. Proc. OCEANS94, Vol. 2, Brest, France, IEEE, 694-699.

_ the LUMINY wind-wave experiment. Gas Transfer and Water Surfaces, Geophys. Monogr., No. 127, Amer. Geophys. Union, 295-301.

__ and Coauthors, 1999: Effects of breaking waves and air-sea gas transfer (LUMINY). Contract ENV4-CT95-0080, TNO Physics and Electronics Laboratory Rep. FEL-99-C122, 178 pp. -, and Coauthors, 2001: LUMINY: An overview. Gas Transfer and Water Surfaces, Geophys. Monogr., No. 127, Amer. Geophys. Union, 291-294.

Duineveld, P. C., 1998: Bouncing and coalescence of bubble pairs at high Reynolds number in pure water or aqueous surfactant solutions. Appl. Sci. Res., 58, 409-439.

Fan, L.-S., and K. Tsuchiya, 1990: Bubble Wake Dynamics in Liquids and Liquid-Solid Suspensions. Butterworth-Heinemann, 363 pp.

Farmer, D. M., C. L. MacNeil, and B. D. Johnson, 1993: Evidence for the importance of bubbles in increasing air-sea gas flux. Nature, 361, 620-623.

Haines, M. A., and B. D. Johnson, 1995: Injected bubble populations in seawater and fresh water measured by a photographic method. J. Geophys. Res., 100 (C4), 7057-7068.

Hwang, P. A., Y.-H. L. Hsu, and J. Wu, 1990: Air bubbles produced by breaking wind waves: A laboratory study. J. Phys. Oceanogr., 20, 19-28.

Jähne, B., and P. Geißler, 1994: An imaging optical technique for bubble measurements. Sea Surface Sound '94. Proceedings of the III International Meeting on Natural Physical Processes Related to Sea Surface Sound, M. J. Buckingham and J. R. Potter, Eds., World Scientific, 290-303.

Johnson, B. D., and R. C. Cooke, 1979: Bubble populations and spectra in coastal waters: A photographic approach. J. Geophys. Res., 84 (C7), 3761-3766.

Kawase, Y., B. Halard, and M. Moo-Young, 1992: Liquid phase mass transfer coefficients in bioreactors. Biotechnol. Bioeng., 39, $1133-1140$.

Kitaigorodskii, S. A., 1984: Wind-wave effects on gas transfer. Gas Transfer at Water Surfaces, W. Brutsaert and G. H. Jirka, Eds., D. Reidel, 141-170.

Leifer, I., 1995: A validation study of bubble mediated air-sea gas transfer modelling. Ph.D. thesis, Georgia Institute of Technology, $176 \mathrm{pp}$.

— wave generated bubble plumes during the LUMINY wind-wave experiment. Gas Transfer and Water Surfaces, Geophys. Monogr., No. 127, Amer. Geophys. Union, 303-309.

_ J. Clark, and R. Chen, 2000a: Modifications of the local environment by a natural marine hydrocarbon seep. Geophys. Res. Lett., 27, 3711-3714.

— G. Ge Leeuw, and L. Cohen, 2000b: Secondary bubble production from breaking waves: The bubble burst mechanism. Geophys. Res. Lett., 27, 4077-4080.

_, R. K. Patro, and P. Bowyer, 2000c: A study on the temperature variation of rise velocity for large clean bubbles. J. Atmos. Oceanic Technol., 17, 1392-1402.

Liss, P. S., and Coauthors, 1997: Physical processes in the microlayer and the air-sea exchange of trace gases. The Sea Surface and Global Change, P. S. Liss and R. A. Duce, Eds., Cambridge University Press, 1-33.

Medwin, H., and N. D. Breitz, 1989: Ambient and transient bubble spectral densities in quiescent seas and under spilling breakers. J. Geophys. Res., 94 (C9), 12 751-12 759.

Monahan, E. C., 1986: The ocean as a source for atmospheric particles. The Role of Air-Sea Exchange in Geochemical Cycling, P. Buat-Menard, Ed., D. Reidel, 129-163.

— , Q. Wang, X. Wang, and M. B. Wilson, 1994: Air entrainment by breaking waves: A laboratory assessment. Proceedings of International Symposium on Aeration Technology, R. E. A. Arndt and A. Prosperetti, Eds., Vol. 187, ASME, 21-26.

NIH Image, 2001: NIH Image Version 1.62b30. U.S. National Institutes of Health. [Available online at http://rsb.info.nih.gov/nihimage.]

Sam, A., C. O. Gomez, and J. A. Finch, 1996: Axial velocity profiles of single bubbles in water/froth. Int. J. Miner. Process., 47, 177196.

Spiel, D., 1998: On the births of film drops from bubbles bursting on seawater surfaces. J. Geophys. Res., 103 (C11), 24 90724918.

Thorpe, S. A., 1982: On the clouds of bubbles formed by breaking wind-waves in deep water and their role in air-sea gas transfer. Philos. Trans. Roy. Soc. London, A304, 155-210.

Uno, S., and R. C. Kintner, 1956: Effect of wall proximity on the rate of rise velocity of single air bubbles in a quiescent liquid. AIChE J., 2, 420-425.

Vagle, S., and D. M. Farmer, 1998: A comparison of four methods for bubble size and void fraction measurements. IEEE J. Oceanic Eng., 23, 211-222.

Walsh, A. L., and P. J. Mulhearn, 1987: Photographic measurements of bubble populations from breaking wind waves at sea. J. Geophys. Res. 92 (C13), 14 553-14 565.

Wang, Q., and E. C. Monahan, 1995: The influence of salinity on the spectra of bubbles formed in breaking wave simulations. Sea Surface Sound '94. Proceedings of the III International Meeting on Natural Physical Processes Related to Sea Surface Sound, M. J. Buckingham and J. R. Potter, Eds., World Scientific, 312319.

Woolf, D. K., 1997: Bubbles and their role in gas exchange. The Sea Surface and Global Change, P. S. Liss and R. A. Duce, Eds., Cambridge University Press, 174-205. 